\title{
Subject Index Vol. 18, 1996
}

Acidosis 515

Adenosine triphosphate 515,535

Adrenal steroids 22

Age polyethism 102

Alanine aminotransferase 505

Alzheimer disease 499

Amino acid transport 484

Amyotrophic lateral sclerosis 492

Anaplerotic and oxidative pathways, TCA

cycle 469 Androgen 124

- receptor 115 Apis mellifera 102 Apteronotus 115 Aspartate 336

- aminotransferase 505 Astrocyte(s) 6, 139,224,255,297,319,

343, 353, 360, 371, 380, 478,484, 515 Astroglia 405 Astroglial cells 397 ATPase 336

Basic fibroblast growth factor 210 Behavioral inhibition 22 Bergmannglia 542 Birdsong 124 Brain 274, 524

- cell culture 443

- development 153

- energy metabolism 542

- metabolism 360 Brain-derived neurotrophic factor 36 Bromodeoxyuridine 199

Bunina bodies 492

Cat 162 cDNA 524 Cell culture 255

- death 297

Central nervous system 243 Cerebellar astrocytes 460 Cerebellum 542 Cerebral gray matter 535

- white matter 535 CG-4 cell line 266 Chemotaxis chamber 266 Compartmentation 380 Confocal microscopy

243 Corpora allata 102

- pedunculata 102 Creatine 524

- kinase 535, 542 Cu,Zn-SOD 397 
Culture 210 Cyclic AMP 224

Cytoskeleton 233

Dentate gyrus 22,199 Deoxyglucose uptake $353\left[{ }^{\mathrm{I} 4} \mathrm{C}\right]$ Deoxyglucose 343 2-Deoxyglucose 336,515 Development 6,139, 243,274, 319,535 Differentiation 255, 353 Dihydrotestosterone 115 DM20 174 Dorsal root ganglion cells 139 Dysmyelination 174,297

Ecdysore receptor 87 Ecdysteroids 87 Electric fish 115

- organ 115

Electrochemical assay 405

Electroconvulsive shock 405

Electron microscopy 210,233

Energy metabolism 371, 380,426,524

Enzyme reactor 405

Epithelial cells, choroid plexus 542 Estrogen 36, 115, 124

- receptors 36

Excitatory input 22

Extracellular fluid 360

4F2 484 Fadrozole 124 Ferritin 274 Fibroblasts 499 Fibronectin 266 Fructose 371

G proteins 153

GABA 434

Galactocerebroside oligodendrocyte 309

Glia 274, 309,434

Glial glucose metabolism 449

Glucocorticoid receptors 49

Glucose 360, 426

$\left[\mathrm{l}^{-13} \mathrm{C}\right]$ Glucose 460

Glucose-6-phosphatase 360

Glutamate 336, 343, 380,434

- metabolism 499

- uptake 515 Glutaminase 224

Glutamine 380,415,434,460,469 Glutathione 391 GlyceroI-3-phosphate 434 
Glycogen 371,405 Glycolysis 515

Granular and pyramidal cells, hippocampus 542 Granule cells 460 - neurons 22 Growth factor receptors 199 p-Guanidinopropionic acid 524

Hippocampus 49,405 HPAaxis 49 Hydrogen peroxide 397 p-Hydroxybutyrate 426 20-Hydroxyecdysone 73 Hypothyroidism, perinatal 153 Hypoxia 443,515

IGF-I 443

Immobilization 478

Immunofluorescence microscopy 542

Immunohistochemistry 492

In situ hybridization 139,309,524

Insulin 443

Intracellular amino acid pools 224

Ion channel 139

Iron 274

Ischemia 405

Isotopomers 460

Jimpy mouse 297

Ketoglutarate metabolism 499 ct-Ketoglutarate dehydrogenase complex

499 Ketoisocaproate 420

Lactate $336,405,443,515$

Laminin 266

Large neutral amino acid transporter 415 ,

420 Leucine 415,420

Lewy body-like hyaline inclusions 492 Lineage 255

Manducasexta 73

Maple syrup urine disease 415,420

Mass isotopomer study of fractional

synthesis 469 Mathematical modelling 460 Memory 6 Merosin 266 Metals 274 Metamorphosis 73 
Methoprene 102 Microcarriers 478 Microdialysis 360,405,415,420 Migration 266 Mitochondria 391,397 Mn-SOD 397 Molecular biology 524 MRI 336 mRNA $139{ }^{13}$ C-MRS 434 Mushroom bodies 102 Myelin 6,243,274,297

- basic protein 233

${ }^{13} \mathrm{~N}$-labeling studies 499

$\mathrm{Na}^{+}$current 115

Na,K transporting ATPase 319

$\mathrm{Na}^{+}, \mathrm{K}^{+}$-ATPase 336, 343

Nerve growth factor 36

Neurodegeneration 391 *

Neurofilaments 210

Neurogenesis 22,199

Neuromuscular development 185

Neuron 360

Neuronal/astrocytic trafficking 391

- differentiation 49

- remodeling 87 Neuron(e)s 255,434,478 Neurosteroids 6 Neurotrophic factors 185 Neurotrophin-3 36 NMDA receptors $22{ }^{, 3}$ C-NMR 449

3.P-NMR 478

Nuclear magnetic resonance 460

- - - spectroscopy 443

0-2A progenitor cell 266,309 Oligodendrocyte(s) 6, 174,255,274,297, 319

- precursor 266 
Organotypic explants 36 Osmotic stress 449 Osteoblast 139 Ouabain 353 Oxidation 224

\section{PC12 cells 36}

Peroxynitrite 391

PET 336

Phaseolus vulgaris leucoagglutinin 162

Phosphocreatine 524,535

Phospholipid biosynthesis 449

Platelet-derived growth factor 266

Postnatal development 426

Potassium 343, 353

Pregnenolone 6

Progesterone 6

Programmed cell death 87

Proteolipid 174

- protein 297

Purkinje neurons 542

Pyridoxal 5'-phosphate-containing enzymes

505 Pyruvate 380

Radial glia 22

Rat central nervous system development 524

- neurons 210

Reactive oxygen species 397

Receptor isoforms 87

Red nucleus 162 Regeneration 6 Retroviral vectors 255 Ribosomes 233 Rubidium 336

S-(1,2-dichlorovinyl)-i>cysteine 505

S-(1,1,2,2-tetrafluoro)-Ircysteine 505 Schwann cells 6 Sensorimotor cortex 162 
Sensory neurons 36

Serial reconstruction 162

Serotonin 49

Sexual differentiation 124

Sexually dimorphic development 185

Skein-like inclusions 492

Social insects 102

Sodium 343

- channel 139 Sorbitol 371 Spinal cord 139,492

- nucleus of bulbocavernosus 185 Sternopygus 115

Stress 22,49, 405 Substance P 115 Sub ventricular region 199 Superoxide dismutase 397,492 Synapse elimination 185 Synaptic competition 185 System L 484

Tenascin-C 266 Testosterone $115\left[{ }^{3} \mathrm{H}\right]$ Thymidine 199 Trafficking 434 Tricarboxylic acid cycle 460 trkA 36 trkB 36 trkC 36

UDP-galactose:ceramide galactosyltrans-ferase 309

Volume regulation 449

Zebra finch 124 
Subject Index Vol. 18,1996 